\title{
Drosophila egg-derived tyrosine phosphatase (EDTP): a novel target for improved survivorship to prolonged anoxia and cellular protein aggregates
}

Short title: Drosophila EDTP and improved survival

Chengfeng Xiao ${ }^{\text {a, }}$, Shuang Qiu ${ }^{\text {a, b }}$, Xiao Li ${ }^{\text {c }}$, Dan-Ju Luo ${ }^{c}$, Gong-Ping Liu ${ }^{\text {c, d, * }}$

a Department of Biology, Queen's University, Kingston, Ontario K7L 3N6 Canada

${ }^{b}$ Jiangsu Key Laboratory of Chemical Pollution Control and Resources Reuse, School of Environmental and Biological Engineering, Nanjing University of Science and Technology, Xiao Ling Wei 200, Nanjing 210094, Jiangsu, P. R. China

${ }^{c}$ Department of Pathophysiology, Key Laboratory of Neurological Diseases of Chinese Ministry of Education, Tongji Medical College, Huazhong University of Science and Technology, Wuhan 430030, P. R. China

${ }^{\mathrm{d}}$ Co-Innovation Center of Neuroregeneration, Nantong University, Nantong JS 226001, P.R. China

* Corresponding authors: xiao.c@queensu.ca (C.X.) and liugp111@ mail.hust.edu.cn (G-P. L.) 


\begin{abstract}
Drosophila egg-derived tyrosine phosphatase (EDTP), a lipid phosphatase that removes 3position phosphate at the inositol ring, has dual functions in the oogenesis and the muscle performance during adult stages. A mammalian homologous gene MTMR14, which encodes the myotubularin-related protein 14, negatively regulates autophagy. Mutation of EDTP/MTMR14, however, causes at least three deleterious consequences: (1) lethality in the early embryogenesis in Drosophila; (2) “jumpy” phenotype with apparently impaired motor functions; and (3) association with a rare genetic disorder called centronuclear myopathy. Here we show that flies carrying a heterozygous EDTP mutation had increased survivorship to prolonged anoxia; tissuespecific downregulation of EDTP in non-muscle tissues, particularly motoneurons, extended the lifespan; and tissue-specific downregulation of EDTP in motoneurons improved the survivorship to beta-amyloid peptides (A $\beta 42)$ and polyglutamine (polyQ) protein aggregates. MTMR14 expression was evident in the hippocampus and cortex in C57BL/6J and APP/PS1 mice. Compared with C57BL/6J mice, APP/PS1 mice had reduced MTMR14 in the cortex but not in the hippocampus. Hippocampal expression of MTMR14 was increased and plateaued at 9-17 months compared with 2-6 months in $\mathrm{C} 57 \mathrm{BL} / 6 \mathrm{~J}$ mice. A $\beta 42$ treatment increased the expression of MTMR14 in the primarily cultured hippocampal neurons of Sprague/Dawley rats and mouse Neuro2a neuroblasts. We demonstrated a novel approach of tissue-specific manipulation of the disease-associated gene EDTP/MTMR14 for lifespan extension and the improvement of survivorship to cellular protein aggregates.
\end{abstract}

\title{
Keywords:
}

Egg-derived tyrosine phosphatase; myotubularin-related protein 14; survival; prolonged anoxia; cellular protein aggregate; tissue-specific expression; motoneuron; Drosophila 


\section{Introduction}

Drosophila egg-derived tyrosine phosphatase (EDTP) is a lipid phosphatase that removes 3position phosphate at the inositol ring of phosphatidylinositol 3-phosphate (PtdIns3P) and phosphatidylinositol $(3,5)$-bi-phosphate $[\operatorname{PtdIns(3,5)P2]~[1].~EDTP~possesses~a~function~opposite~}$ to Vps34, a sole class III phosphoinositide 3-kinase [2,3], in the regulation of the PtdIns3P pool. The most interesting characteristic of EDTP expression is that there are two peaks, one at oogenesis [4,5], and another at adult stages [6,7]. The transcription of MTMR14, a mouse homolog of EDTP, also shows a peak at day five of differentiation in $\mathrm{C} 2 \mathrm{C} 12$ myoblasts, followed by a decline [1]. Human MTMR14 transcripts are detectable at the ages between 19 and 69 years in the tested tissues, including heart, brain, placenta, lung, liver, skeletal muscle, kidney, and pancreas [1]. Levels of MTMR14 are highly coincident with those of Vps34 in the human brain, heart, and skeletal muscles [8], indicating a tightly regulated biological process which is associated with cellular PtdIns3P levels.

The decline of EDTP between early embryogenesis and young adult stages is accompanied with the Drosophila metamorphosis, a process requiring extensive autophagy and apoptosis for histolysis [9]. These observations suggest a role for EDTP in the regulation of autophagy. This is indeed supported by the findings that PtdIns3P stimulates autophagy in human HT-29 cells [10], that MTMR14 negatively controls the autophagosome formation and maturation in mammalian cells [11], and that the EDTP/MTMR14 inhibitor, AUTEN-99, activates autophagy in human HeLa cells and mouse tissues [12]. Interestingly, the latter group also shows that overexpression of a modified EDTP (by skipping the first exon) in the fat body antagonizes the effect of AUTEN-67 in inducing autophagy [13]. 
Despite the negative regulation of autophagy, the deletion of Drosophila EDTP is lethal during embryogenesis or in the first instar, and germline clones with a null EDTP allele fail to produce mature oocytes [5]. Homozygous flies carrying a hypomorphic EDTP allele are shortlived with impaired motor functions and reduced fecundity [14]. Additionally, muscles of the MTMR14-deficient mice have decreased force production, prolonged relaxation and exacerbated fatigue [15]. A human MTMR14 missense variant (R336Q) is associated with centronuclear myopathy, a rare genetic disorder with muscle weakness and wasting [1]. Therefore, the function of autophagy initiation is likely overwhelmed by the lethality or disease-causing effects due to a ubiquitous loss of EDTP/MTMR14.

There are advantages of autophagy in degrading and recycling disrupted organelles, longlived proteins, and denatured protein aggregates [10,16]. A strategy to maximize the potential benefit of EDTP is to manipulate its downregulation in the favorable tissues while leaving the expression intact in oocytes and as well as in muscles at adult stages. This seems to be feasible by using the Drosophila Gal4/UAS expression system [17]. We thus hypothesized that selective downregulation of EDTP in non-muscle tissues, particularly the central nervous system, extends lifespan and improves the survivorship to cellular protein aggregates in Drosophila.

In the current study, we demonstrate that heterozygous EDTP mutants had improved survival to prolonged anoxia, a condition mimicking the extreme hypoxia that induces autophagy in mammalian and human cells [18]. We also show that selective downregulation of EDTP in the fly motoneurons extended lifespan and increased the survivorship to beta-amyloid peptides (A 342 ) or polyglutamine (polyQ) protein aggregates. The expression of MTMR14 in the hippocampus and cortex in mice was evident, promising a potential application by targeting EDTP/MTMR14 for the treatment of neurodegeneration. 


\section{Materials and methods}

\section{Animals}

Fly strains used in this study and their sources were: EDTP DJ694 (FBal0160586) and $w^{1118}(\mathrm{~L}$. Seroude laboratory, Queen's University), D42-Gal4 (Bloomington Drosophila Stock Center (BDSC) \#8816), UAS-EDTP-RNAi (II) (with an RNAi sequence CAGTAGTGTAATAGTAATCAA targeting exon 3 of EDTP, FBst0041633), UAS-EDTPRNAi (III) (with an RNAi sequence CAGCTACGACGAAGTCATCAA targeting exon 2, FBst0036917), UAS-Aß42 (BDSC \#32038), UAS-Httex1-Q103-eGFP [19]. Flies were maintained with standard medium (cornmeal, agar, molasses, and yeast) at $21-23{ }^{\circ} \mathrm{C}$ with a 12h:12h photoperiod.

Heterozygous mutants $\left(E D T P^{D J 694} /+\right)$ and their sibling controls $\left(w^{1118}\right)$ were prepared by two consecutive crosses between homozygous $E D T P^{D J 694}$ mutants and $w^{1118}$ flies. We chose virgin female offspring $\left(E D T P^{D J 694} /+\right)$ from the first mating and crossed them with $w^{1118}$ males. Flies carrying the EDTP mutation (red-eyed) and their siblings (white-eyed) were collected for survival analysis.

C57BL/6J mice and Sprague/Dawley rats were purchased from the Experimental Animal Centre of Wuhan University. The C57BL/6J mice are wild-type whereas APP/PS1 (stock number 004462, Jackson Laboratory) are the transgenic mouse models of Alzheimer's disease. The APP/PS1 mice express a chimeric mouse/human amyloid precursor protein (Mo/HuAPP695swe) and a mutant human presenilin 1 (PS1-dE9) both directed to CNS neurons. The mice were housed in a light/dark (12h:12h) cycle in standard group cages (4-5 per cage) 
with accessible food and water ad libitum. The Sprague/Dawley rats were used for the isolation of primary hippocampal neurons for cell culture. Experimental procedures with mice were approved by the Ethics Committee of Tongji Medical College, Huazhong University of Science and Technology.

\section{Post-anoxia survival}

Newly emerged flies were collected and aged to 6-8 days in regular culture conditions and exposed to an anoxia (generated by pure argon gas) during the light phase of the photoperiod. The percent survival at $12 \mathrm{~h}$ (\% 12-h survival) after anoxia was examined. Replicated groups (15-25 flies per group, $\mathrm{n}=3 \sim 7$ ) of flies were examined. We chose the anoxia exposure of 1,3 , or $6 \mathrm{~h}$ because flies are highly tolerant to anoxia for hours [20].

For the tests of post-anoxia long-term survivorship, flies were exposed to anoxia for 0 or $6 \mathrm{~h}$. The dead flies were scored daily during the first week of recovery, and twice a week thereafter until all the flies were counted. Throughout the long-term survival experiments, alive flies were transferred to fresh food vials twice a week.

\section{Lifespan experiments}

Newly emerged flies were collected and raised at a density of 20-25 flies per vial. They were transferred into fresh culture media twice a week. The lifespan was scored by counting dead flies during each transfer until all the flies were counted. This procedure was also used for the examination of the lifespan of flies expressing $\beta$ amyloid $(A \beta)$ peptides N-42 (A $\beta 42)$ or polyglutamine (polyQ) protein aggregates (with 103 tandem repeats) in motoneurons, and the 
lifespan of flies with simultaneous expression of A $\beta 42$ or polyQ aggregates and RNAi knockdown of EDTP in the same cells. Only male flies were tested.

\section{Cell Culture}

The rat primary hippocampal neurons were prepared by following a reported method [21]. Briefly, hippocampal neurons from the 18-day-old embryonic (E18) Sprague/Dawley rats were isolated and seeded at 30,000 - 40,000 cells per well on 6-well plates coated with Poly-DLysine/Laminin (Bioscience) in the neurobasal medium (Invitrogen), which was supplemented with $2 \%$ B27/0.5 mM glutamine/25 mM glutamate. Half of the culture medium was changed every 3 days with neurobasal medium supplemented with 2\% B27 and $0.5 \mathrm{mM}$ glutamine. All cultures were kept at $37^{\circ} \mathrm{C}$ in a humidified $5 \% \mathrm{CO}_{2}$ cultural condition.

Neuro2a cells were cultured at $37^{\circ} \mathrm{C}$ in a 1:1 mixture of DMEM (Dulbecco's modified Eagle's medium) and OPTI-MEM supplemented with 15\% FBS (fetal bovine serum, Gibco), 100 units $/ \mathrm{ml}$ penicillin, and $100 \mathrm{mg} / \mathrm{ml}$ streptomycin. A humidified atmosphere containing $5 \% \mathrm{CO}_{2}$ was provided. The cells were plated on to six-well plates overnight and treated with $5 \mu \mathrm{M}$ A $\beta 42$ or vehicle control (DMSO) for $24 \mathrm{~h}$.

\section{Immunohistochemistry}

Mice were anesthetized and perfused through aorta with $0.9 \% \mathrm{NaCl}$ followed by phosphate buffer containing $4 \%$ paraformaldehyde. The brains were removed and post-fixed in perfusate overnight. Tissue sectioning $(20 \mu \mathrm{m})$ was performed with a vibratome (Leica Biosystems). The sections were fixed with $0.3 \% \mathrm{H}_{2} \mathrm{O}_{2}$ in the absolute methanol for $30 \mathrm{~min}$ and saturated with 
bovine serum albumin (BSA) for $30 \mathrm{~min}$ at room temperature. Tissues were then incubated with polyclonal anti-MTMR14 overnight at $4{ }^{\circ} \mathrm{C}$. After wash, the tissues were incubated with appropriate secondary antibody. Immunoreaction was developed with diaminobenzidine using Histostain-SP Kits (Zymed, CA, USA). Images were taken using a light microscope (Olympus BX60, Tokyo, Japan).

\section{Western Blotting}

Western blotting was performed as described previously [22]. The hippocampal region and cortex were quickly removed and homogenized in the sample buffer (Tris-HCl 50mM (pH 7.4), $\mathrm{NaCl} 150$ mM, NaF 10mM, Na3VO4 1mM, EDTA 5 mM, benzamidine 2 mM, and phenylmethylsulphonyl fluoride $1 \mathrm{mM})$. The extracts were mixed with sample buffer $(3: 1, \mathrm{v} / \mathrm{v}$, containing $200 \mathrm{mM}$ Tris-HCl (pH 7.6), 8\% SDS, 40\% glycerol, 40mM dithiothreitol) and incubated with boiled water for $10 \mathrm{~min}$. The proteins were separated by $10 \%$ SDSpolyacrylamide gel electrophoresis and transferred to nitrocellulose membrane. The membrane was blocked for $1 \mathrm{~h}$ with 5\% skim milk (dissolved in TBSTween-20 containing 50mM Tris-HCl (pH 7.6), $150 \mathrm{mM} \mathrm{NaCl}, 0.2 \%$ Tween-20), and probed with primary antibody at $4{ }^{\circ} \mathrm{C}$ overnight. Blots were incubated with anti-rabbit or anti-mouse IgG conjugated to IRDye (800 CW) at room temperature and visualized using the Odyssey Infrared Imaging System (LI-COR Biosciences, Lincoln, NE, USA). Protein bands were analyzed using ImageJ [23].

\section{Statistics}


Statistical analysis was conducted by using R [24] and the following packages: gdata, survival, survminer, and ggplot2. A two-way ANOVA with Tukey's test was applied for the analysis of 12-h survival of different genotypes of flies which were subject to anoxia with varying durations. A log-rank test was performed for the comparison of two survival curves. A log-rank test with the Benjamini - Hochberg ("BH") adjustment was used for the comparison of three survival curves. One-way ANOVA with Bonferroni's multiple comparisons was performed to analyze the MTMR14 expression among three groups of cells. A student $t$-Test was conducted to compare the levels of MTMR14 between two groups of cells. A value of $P<0.05$ was considered statistical significance.

\section{Results}

\section{Improved 12-h survival to prolonged anoxia in male flies of EDTP mutant}

$E D T P^{D J 694}$ is an enhancer-trap line carrying a transposable element $P\{G a w B\}$ in the first intron with the orientation opposite to the affected EDTP gene [6,7]. Flies homozygous for $E D T P^{D J 694}$ are hypomorphic (reduced EDTP transcription, short-lived, motor defective, and reduced fecundity) [14]. To avoid the potential confounding effect, we started to examine the flies heterozygous for $E D T P^{D J 694}$ allele.

$E D T P^{D J 694} /+$ flies and their sibling controls $\left(w^{1118}\right)$ were raised to 6-8 days old and exposed to an anoxia (generated by pure argon gas) for 1,3 , or $6 \mathrm{~h}$. After $1 \mathrm{~h}$ anoxia, the average percentage of survival at $12 \mathrm{~h}(\%$ 12-h survival) of male flies was $100.0 \pm 0.0 \%$ (Mean $\pm \mathrm{SD})$ in both $E D T P^{D J 694} /+$ and controls (Fig. 1a). With 3 h exposure, EDTP $P^{D J 694} /+$ males showed the average \% 12-h survival $(83.3 \pm 10.8 \%)$ higher than controls $(67.9 \pm 8.4 \%)(P=0.0097$, Two- 
way ANOVA with Tukey's test). After $6 \mathrm{~h}$ anoxia, EDTP ${ }^{D J 694} /+$ males had the $\% 12$-h survival in $(63.4 \pm 6.7 \%)$ remarkably higher than controls $(28.4 \pm 5.2 \%)(P<0.0001$, Two-way ANOVA with Tukey's test). The genotype, anoxia duration, and their interaction were the factors all significantly contributed to the \% 12-h survival in male flies (Two-way ANOVA) (Fig. 1b). Female EDTP ${ }^{D J 694} /+$ flies had the $\%$ 12-h survival statistically the same as $w^{1118}$ flies with any of the 1, 3, or $6 \mathrm{~h}$ exposure. Two-way ANOVA indicated that while anoxia duration significantly contributed to \% 12-h survival, the genotype or the interaction factor had an insignificant contribution (Fig. 1b). Therefore, male EDTP ${ }^{D J 694} /+$ flies displayed improved 12-h survival to the prolonged anoxia (i.e. 3 - 6 h duration), whereas both $E D T P^{D J 694} /+$ and control females had comparably enhanced 12-h survival to the prolonged anoxia.

\section{Increased post-exposure survivorship to prolonged anoxia in EDTP mutant}

The increased 12-h survival to prolonged anoxia raised an immediate question: whether EDTP mutants have increased post-exposure survivorship throughout the life. Young flies (6-8 days old) were exposed to a 6-h anoxia and the post-exposure survivorship was examined. We used the 6-h exposure because the \% 12-h survival was markedly different between heterozygous mutant and controls.

Without anoxia, the median survival time was 69 d (95\% confidence interval (CI) 65 - 69 d, $\mathrm{n}=102)$ in $E D T P^{D J 694} /+$ males and $69 \mathrm{~d}(\mathrm{CI} 65-69 \mathrm{~d}, \mathrm{n}=96)$ in controls. There was no statistical difference in the survivorship between these groups ( $P=0.945$, Log-rank test) (Fig. 2). There was also no significant difference in the survivorship between female $E D T P^{D J 694} /+$ (median $74 \mathrm{~d}, \mathrm{CI} 69-79 \mathrm{~d}, \mathrm{n}=107)$ and their controls (median $74 \mathrm{~d}, \mathrm{CI} 69-79 \mathrm{~d}, \mathrm{n}=97)(P=$ 
0.869, Log-rank test). Thus, without anoxia, the EDTP mutation displayed no effect on the survivorship.

With $6 \mathrm{~h}$ anoxia, EDTP ${ }^{D J 694} /+$ males showed post-exposure survival (median 36 d, CI 1 - 72 $\mathrm{d}, \mathrm{n}=167)$ markedly longer than controls (median $1 \mathrm{~d}, \mathrm{CI} 1-1 \mathrm{~d}, \mathrm{n}=163)(P=0.0033$, Logrank test). $E D T P^{D J 694} /+$ females also showed the post-exposure survival (median 63.5 d, CI 37 $79 \mathrm{~d}, \mathrm{n}=150)$ remarkably longer than controls (median $11 \mathrm{~d}, \mathrm{CI} 8-37 \mathrm{~d}, \mathrm{n}=148)(P=0.0001$, Log-rank test). Both male and female flies of $E D T P^{D J 694} /+$ had increased post-exposure survivorship to $6 \mathrm{~h}$ anoxia.

\section{RNAi knockdown of EDTP in motoneurons extended lifespan}

Extreme hypoxia induces autophagy in mammalian and human cells [18]. The mammalian homologous MTMR14 negatively regulates the autophagic removal of protein aggregates [11]. These data together with our findings suggested that downregulation of EDTP could promote autophagy and improve the survivorship. We used the Gal4/UAS binary system [17] for selective downregulation of EDTP in motoneurons and examined the lifespan. The rationale for targeting motoneurons was to avoid affecting the abundant expression of EDTP in muscles, ovaries, spermatheca, and oocytes. The motoneuron-specific driver D42-Gal4 [25] was used for RNAi knockdown.

We used the UAS-EDTP-RNAi (III) line with a short interference sequence targeting the transcripts from exon 2 of EDTP. Flies with EDTP knockdown in motoneurons (D42-Ga14/UASEDTP-RNAi (III)) had a median lifespan of $113 \mathrm{~d}(\mathrm{CI} 107-118 \mathrm{~d}, \mathrm{n}=132)$, which was longer than UAS controls (UAS-EDTP-RNAi (III)/+, median 89 d, CI $89-98$ d, n = 63) $(P<0.0001$, 
Log-rank test with "BH" adjustment), and also longer than Gal4 controls (D42-Gal4/+, median

73 d, CI $73-77$ d, n = 137) $(P<0.0001$, Log-rank test with "BH” adjustment) (Fig. 3a).

Therefore, downregulation of EDTP in motoneurons resulted in extended lifespan.

To confirm this finding, we used a different RNAi line (UAS-EDTP-RNAi (II) with an interference sequence targeting the transcripts from exon 3). Flies with EDTP knockdown in motoneurons (UAS-EDTP-RNAi (II)/+; D42-Gal4/+) had the lifespan (median 74.5 d, CI 69 $84 \mathrm{~d}, \mathrm{n}=114)$ significantly longer than UAS controls (median $58 \mathrm{~d}$, CI $51-58 \mathrm{~d}, \mathrm{n}=79)(P<$ 0.0001, Log-rank test with "BH" adjustment), or Gal4 controls (median $51 \mathrm{~d}, \mathrm{CI} 51-54 \mathrm{~d}, \mathrm{n}=$ 87) $(P<0.0001$, Log-rank test with "BH" adjustment) (Fig. 3b). These data indicated that downregulation of EDTP in motoneurons extended the lifespan in Drosophila.

\section{EDTP knockdown in motoneurons improved the survivorship to beta-amyloid peptides or}

\section{polyglutamine protein aggregates}

We next asked whether downregulation of EDTP could increase the survivorship to cellular protein aggregates expressed in the motoneurons. We examined the survivorship of flies with simultaneous EDTP downregulation and the expression of toxic $\beta$ amyloid (A $\beta$ ) peptides N-42 (A $\beta 42)$ [26]. The median lifespan in flies expressing A $\beta 42$ in motoneurons (UAS-A $\beta 42 / Y ;$; $42-$ Gal4/+) was $64 \mathrm{~d}(\mathrm{CI} 64-64 \mathrm{~d}, \mathrm{n}=111)$. The median lifespan was increased to $94 \mathrm{~d}(\mathrm{CI} 94$ - 98 $\mathrm{d}, \mathrm{n}=86$ ) in flies with A $\beta 42$ expression and simultaneous RNAi knockdown of EDTP (UASAß42/Y;; D42-Gal4/UAS-EDTP-RNAi) $(P<0.0001$, Log-rank test) (Fig. 4a).

We also examined the survivorship of flies with EDTP downregulation and simultaneous expression of polyglutamine (polyQ) protein aggregates in the motoneurons. The median 
lifespan was 38 d (CI 38 - 38 d, n = 45) in flies (UAS-Httex1-Q103-eGFP/Y;; D42-Gal4/+)

expressing polyQ aggregates (each containing a polyQ tract of 103 tandem repeats of glutamine).

The median lifespan was significantly increased to $44.5 \mathrm{~d}(\mathrm{CI} 41-48 \mathrm{~d}, \mathrm{n}=30)$ in flies (UASHttex1-Q103-eGFP/Y;; D42-Gal4/UAS-EDTP-RNAi) with both polyQ expression and EDTP downregulation in motoneurons $(P<0.0001$, Log-rank test) (Fig. 4b).

Therefore, EDTP downregulation in motoneurons improved the survivorship to the expression of $A \beta 42$ or polyQ protein aggregates.

\section{Expression of MTMR14 in the hippocampus and cortex in C57BL/6J and APP/PS1 mice}

Human MTMR14 is transcribed ubiquitously at relatively low levels in the brain [27]. Mouse MTMR14 is expressed in the muscles, liver, and fat but there is little evidence for the brain expression [28]. We examined the expression of MTMR14 in the hippocampus and cortex in C57BL/6J and APP/PS1 mice. C57BL/6J is wild-type, whereas APP/PS1 is a transgenic strain with constitutive expression of a chimeric mouse/human amyloid precursor protein (Mo/HuAPP695swe) and a mutant human presenilin 1 (PS1-dE9) in CNS neurons [29].

MTMR14 expression was observable in the CA3 pyramidal neurons and dentate gyrus (DG) in the hippocampus of a 3-month-old C57BL/6J mouse (Fig. 5a). MTMR14 expression was detectable in the hippocampus in both C57BL/6J and APP/PS1 mice. The levels in two strains were similar with no significant difference (Fig. 5b). MTMR14 expression was also detectable in the cortex in both strains. However, relative levels of MTMR14 in APP/PS1 mice were lower than those in $\mathrm{C} 57 \mathrm{BL} / 6 \mathrm{~J}$ mice $(P<0.05, t$-Test). These data indicated an association between the transgenic expression of amyloid precursor protein/presenilin 1 and MTMR14 level. 
Relative levels of MTMR14 in the C57BL/6J hippocampus increased and plateaued at 9, 12 and 17 months compared with the levels at 2 and 6 months (Fig. 5c), suggesting an agedependent kinetics of MTMR14 expression.

\section{Increased MTMR14 induced by A 442 in the rat primary hippocampal neurons and}

\section{Neuro2a cells}

A 342 causes microRNA deregulation in primary hippocampal neurons in C57BL/6J mice [30] and inhibits the viability of the mouse neuroblastoma Neuro2a cells [31]. We explored the effect of A 342 treatment on MTMR14 expression in primary hippocampal neurons and Neuro2a cells.

The primary hippocampal neurons from 18-day-old embryonic (E18) Sprague/Dawley rats were prepared and treated with A $\beta 42$. Relative MTMR14 levels were increased in the primary cultures after a 72-h incubation with $10 \mu \mathrm{M}$ A $\beta 42$ compared with controls $(P<0.01$, One-way ANOVA with Bonferroni's multiple comparisons) (Fig. 6a). There was no significant increase of relative MTMR14 levels in the cultures incubated with $5 \mu \mathrm{M} A \beta 42$ for $72 \mathrm{~h}$. Data indicated a dosage-dependent induction of MTMR14 by A $\beta 42$ treatment. We examined the MTMR14 expression in Neuro2a cells with A $\beta 42$ treatment. MTMR14 expression was increased in Neuro2a cells treated with $\mathrm{A} \beta 42$ at $5 \mu \mathrm{M}$ for $24 \mathrm{~h}$ compared with controls $(P<0.01, t$-Test) (Fig. 6b). Therefore, A $\beta 42$ treatment increased the expression of MTMR14 in both the primary hippocampal neurons of rats and Neuro2a cells.

\section{Discussion}


We report in Drosophila a novel approach of fine-tuning the disease-associated gene EDTP/MTMR14 for lifespan extension and improved survivorship to cellular protein aggregates. Cell-specific downregulation of EDTP in non-muscle tissues circumvents the deleterious consequences of ubiquitous loss of EDTP. Specifically, downregulation of EDTP in motoneurons extends lifespan and increases the survivorship to A $\beta 42$ or polyQ protein aggregates. The expression of the mouse MTMR14 in the hippocampus and cortex, together with its age-dependent kinetics and the A $\beta 42$-induced increase of MTMR14 in the rat's cultured hippocampal neurons, promises a potential application by targeting EDTP/MTMR14 in a tissuespecific manner for the treatment of neurodegeneration.

The proposed cell-specific downregulation of EDTP/MTMR14 for beneficial consequences is formulated largely from the preliminary observation that heterozygous flies carrying the $E D T P^{D J 694}$ allele have increased survivorship to prolonged anoxia. Homozygous flies carrying this allele display a "jumpy" phenotype - impaired motor function associated with shortened lifespan and reduced fecundity [14]. These findings indicate that downregulation of EDTP in flies homozygous for $E D T P^{D J 694}$ is overall deleterious, whereas moderate downregulation of EDTP in heterozygous flies causes no obvious motor defect and becomes protective if flies are exposed to a prolonged anoxia. Such a conditional benefit highlights the importance of subtle manipulation of spatiotemporal expression of EDTP/MTMR14 for the desired effect.

EDTP/MTMR14 has two main functions: negative regulation of autophagy and disease-causing effect if deficient in the muscles [1,11-15]. Drosophila EDTP has two peaks of expression, one at oogenesis and another at adult stages around 20-30 days [4-7]. Both male and female adult flies have abundant EDTP in muscles and additionally, female flies express rich amounts of EDTP in the spermatheca and ovaries. The differential expression of EDTP in female flies might 
also be responsible for the enhanced short-term survival (i.e. \% 12-h survival) to prolonged anoxia. The dual functions, multiple peaks over time, broad expressing tissues, and the sexual dimorphism of expression pattern together do not likely allow a complete deletion of EDTP/MTMR14 to be associated with beneficial consequences. Precise control of EDTP/MTMR14 expression in the favorable cells or tissues could be essential for eliciting the protective effects. The current study presents a strategy of cell-specific downregulation of Drosophila EDTP in motoneurons with beneficial effects in lifespan extension and improved survivorship to cellular protein aggregates.

Motoneurons are highly specialized and terminally differentiated with reduced function of global genome repair [32,33]. More importantly, neuronal cells utilize autophagy as an essential process for normal turnover of cytoplasmic contents [34]. Drosophila motoneuronal expression of a human $\mathrm{Cu}-\mathrm{Zn}$ superoxide dismutase (SOD1) leads to a marked extension of lifespan by up to $40 \%$ [35]. Motoneuronal overexpression of the heat shock protein 70 results in structural plasticity of axonal terminals which is associated with increased larval thermotolerance [36]. Motoneurons innervate muscle cells through neuromuscular junctions, making them an ideal target for EDTP downregulation while leaving muscular expression intact. The findings that motoneuronal downregulation of EDTP extends lifespan and improves the survivorship to A $\beta 42$ or polyQ protein aggregates firmly support motoneurons as a favorable targeting tissue. Notably, our findings greatly rely on the putative motoneuronal driver D42-Gal4, which also shows a prominent expression pattern in the peripheral sensory neurons [37]. Whether sensory neurons utilize the EDTP/MTMR14-associated autophagy for removing cellular wastes is unclear, but a study has shown the connection between the sensory neurons and lifespan extension [38]. 
EDTP/MTMR14 possesses a function opposite to Vps34 in the regulation of the PtdIns3P pool. Vps34 is the only phosphatidylinositol 3-kinases in yeast [2] and has been evolutionarily conserved through mammals. Vps34 plays an essential role in the process of autophagy $[39,40]$. Downregulation of EDTP/MTMR14 shifts the focus from Vps34 to its functional opponent for the regulation of PtdIns3P and thus represents a novel approach to manipulate the process of autophagy.

The evident expression of MTMR14 in the mouse hippocampus and cortex provides a foundation from which the cell-specific manipulation of EDTP/MTMR14 expression could be applied in mammals. Furthermore, the transgenic expression of a chimeric mouse/human amyloid precursor protein (Mo/HuAPP695swe) and a mutant human presenilin 1 (PS1-dE9) in APP/PS1 mice is associated with the downregulation of MTMR14 in the cortex but not in the hippocampus. These findings indicate an involvement of MTMR14 expression in the deposit of amyloid peptides and this involvement is tissue-specific. Moreover, MTMR14 expression displays an age-related kinetics and MTMR14 is inducible by A $\beta 42$ treatment in the rat primary hippocampal neurons as well as mouse Neuro2a cells, suggesting a close relation between MTMR14 expression and aging. Noteworthy, MTMR14 expression in response to the presence of amyloid peptides is different between in vivo animal experiments and cultured neurons. Perhaps the increase of MTMR14 in cultured neurons represents a short-term response to A $\beta 42$, whereas MTMR14 downregulation in the animal studies is involved in an active process for the removal of amyloid peptides. Nevertheless, our findings highlight a promising application through targeting EDTP/MTMR14 for lifespan extension and survival improvement to cellular protein aggregates in mammals. 


\section{Conflict of interest}

All authors declare no competing interests.

\section{Acknowledgments}

This study was funded by the Fundamental Research Funds for the Central Universities from

Nanjing University of Science and Technology (30918011308) and Natural Science Foundation of China (81501211 to D.J. L.).

\section{References}

[1] Tosch V, Rohde HM, Tronchère H, et al. A novel PtdIns3P and PtdIns $(3,5)$ P2 phosphatase with an inactivating variant in centronuclear myopathy. Hum Mol Genet. 2006;15:3098-3106.

[2] Schu P, Takegawa K, Fry M, et al. Phosphatidylinositol 3-kinase encoded by yeast VPS34 gene essential for protein sorting. Science (80-. ). [Internet]. 1993;260:88-91. Available from: http://www.sciencemag.org/cgi/doi/10.1126/science.8385367.

[3] Hiles ID, Otsu M, Volinia S, et al. Phosphatidylinositol 3-kinase: structure and expression of the $110 \mathrm{kd}$ catalytic subunit. Cell. 1992;70:419-429.

[4] Krauchunas AR, Horner VL, Wolfner MF. Protein phosphorylation changes reveal new candidates in the regulation of egg activation and early embryogenesis in D. melanogaster. Dev Biol. 2012;370:125-134.

[5] Yamaguchi S, Katagiri S, Sekimizu K, et al. Involvement of EDTP, an egg-derived 
tyrosine phosphatase, in the early development of Drosophila melanogaster. J. Biochem. $2005 ; 138: 721-728$.

[6] Seroude L, Brummel T, Kapahi P, et al. Spatio-temporal analysis of gene expression during aging in Drosophila melanogaster. Aging Cell [Internet]. 2002;1:47-56. Available from: http://doi.wiley.com/10.1046/j.1474-9728.2002.00007.x.

[7] Singh SH, Ramachandra NB, Nongthomba U. Egg-derived tyrosine phosphatase as a potential biomarker for muscle ageing and degeneration in Drosophila melanogaster. J. Genet. Genomics. 2014. p. 221-224.

[8] Volinia S, Dhand R, Vanhaesebroeck B, et al. A human phosphatidylinositol 3-kinase complex related to the yeast Vps34p-Vps15p protein sorting system. EMBO J. $1995 ; 14: 3339$.

[9] Zirin J, Cheng D, Dhanyasi N, et al. Ecdysone signaling at metamorphosis triggers apoptosis of Drosophila abdominal muscles. Dev. Biol. [Internet]. 2013;383:275-284. Available from: http://dx.doi.org/10.1016/j.ydbio.2013.08.029.

[10] Petiot A, Ogier-Denis E, Blommaart EFC, et al. Distinct classes of phosphatidylinositol 3'-kinases are involved in signaling pathways that control macroautophagy in HT-29 cells. J. Biol. Chem. [Internet]. 2000;275:992-998. Available from: http://www.jbc.org/cgi/doi/10.1074/jbc.275.2.992.

[11] Vergne I, Roberts E, Elmaoued RA, et al. Control of autophagy initiation by phosphoinositide 3-phosphatase jumpy. EMBO J. [Internet]. 2009;28:2244-2258. Available from: http://emboj.embopress.org/cgi/doi/10.1038/emboj.2009.159.

[12] Kovács T, Billes V, Komlós M, et al. The small molecule AUTEN-99 (autophagy 
enhancer-99) prevents the progression of neurodegenerative symptoms. Sci Rep. 2017;7.

[13] Papp D, Kovács T, Billes V, et al. AUTEN-67, an autophagy-enhancing drug candidate with potent antiaging and neuroprotective effects. Autophagy. 2016;12:273-286.

[14] Edelman SW. Molecular Characterisation of the Age-regulated Jumpy Gene in Drosophila melanogaster. Library and Archives Canada= Bibliothèque et Archives Canada; 2005.

[15] Shen J, Yu W-M, Brotto M, et al. Deficiency of MIP/MTMR14 phosphatase induces a muscle disorder by disrupting Ca2+ homeostasis. Nat Cell Biol. 2009;11:769-776.

[16] Takeshige K, Baba M, Tsuboi S, et al. Autophagy in yeast demonstrated with proteinasedeficient mutants and conditions for its induction. J Cell Biol. 1992;119:301.

[17] Brand AH, Perrimon N. Targeted gene expression as a means of altering cell fates and generating dominant phenotypes. Development. 1993;118:401-415.

[18] Bellot G, Garcia-Medina R, Gounon P, et al. Hypoxia-induced autophagy is mediated through hypoxia-inducible factor induction of BNIP3 and BNIP3L via their BH3 domains. Mol Cell Biol. 2009;29:2570-2581.

[19] Zhang S, Binari R, Zhou R, et al. A genomewide RNA interference screen for modifiers of aggregates formation by mutant huntingtin in Drosophila. Genetics. 2010;184:11651179.

[20] Callier V, Hand SC, Campbell JB, et al. Developmental changes in hypoxic exposure and responses to anoxia in Drosophila melanogaster. J Exp Biol. 2015;218:2927-2934.

[21] Luo Y, Nie Y-J, Shi H-R, et al. PTPA activates protein phosphatase-2A through reducing its phosphorylation at tyrosine-307 with upregulation of protein tyrosine phosphatase 1B. Biochim. Biophys. Acta - Mol. Cell Res. [Internet]. 2013;1833:1235-1243. Available 
from: http://linkinghub.elsevier.com/retrieve/pii/S0167488913000529.

[22] Duan D-X, Chai G-S, Ni Z-F, et al. Phosphorylation of tau by death-associated protein kinase 1 antagonizes the kinase-induced cell apoptosis. J. Alzheimers. Dis. [Internet]. 2013;37:795-808. Available from: http://www.ncbi.nlm.nih.gov/pubmed/23948915.

[23] Schneider CA, Rasband WS, Eliceiri KW. NIH Image to ImageJ: 25 years of image analysis. Nat. Methods [Internet]. 2012;9:671-675. Available from: http://www.nature.com/doifinder/10.1038/nmeth.2089.

[24] R Development Core Team. R: A Language and Environment for Statistical Computing. R Found. Stat. Comput. Vienna Austria [Internet]. 2016;0:\{ISBN\} 3-900051-07-0. Available from: http://www.r-project.org/.

[25] Yeh E, Gustafson K, Boulianne GL. Green fluorescent protein as a vital marker and reporter of gene expression in Drosophila. Proc Natl Acad Sci U A S. 1995;92:70367040.

[26] Kuo YM, Emmerling MR, Vigo-Pelfrey C, et al. Water-soluble Abeta (N-40, N-42) oligomers in normal and Alzheimer disease brains. J. Biol. Chem. [Internet]. 1996;271:4077-4081. Available from: http://www.jbc.org/content/271/8/4077\%5Cnhttp://www.jbc.org/content/271/8/4077.full.p df\%5Cnhttp://www.jbc.org/content/271/8/4077.long\%5Cnhttp://www.ncbi.nlm.nih.gov/p ubmed/8626743.

[27] Fagerberg L, Hallström BM, Oksvold P, et al. Analysis of the human tissue-specific expression by genome-wide integration of transcriptomics and antibody-based proteomics. Mol. Cell. Proteomics [Internet]. 2014;13:397-406. Available from: 
http://www.mcponline.org/lookup/doi/10.1074/mcp.M113.035600.

[28] Lv Y, Xue L, Cai C, et al. Deficiency of myotubularin-related protein 14 influences body weight, metabolism, and inflammation in an age-dependent manner. Cell Biosci. 2015;5.

[29] Jankowsky JL, Fadale DJ, Anderson J, et al. Mutant presenilins specifically elevate the levels of the 42 residue $\beta$-amyloid peptide in vivo: evidence for augmentation of a 42 specific $\gamma$ secretase. Hum. Mol. Genet. [Internet]. 2004;13:159-170. Available from: https://academic.oup.com/hmg/article-lookup/doi/10.1093/hmg/ddh019.

[30] Schonrock N, Ke YD, Humphreys D, et al. Neuronal MicroRNA Deregulation in Response to Alzheimer's Disease Amyloid- $\beta$. Feany MB, editor. PLoS One [Internet]. 2010;5:e11070. Available from: http://dx.plos.org/10.1371/journal.pone.0011070.

[31] Dahlgren KN, Manelli AM, Stine WB, et al. Oligomeric and Fibrillar Species of Amyloid$\beta$ Peptides Differentially Affect Neuronal Viability. J. Biol. Chem. [Internet]. 2002;277:32046-32053. Available from: http://www.ncbi.nlm.nih.gov/pubmed/12058030.

[32] Aranda-Anzaldo A. The post-mitotic state in neurons correlates with a stable nuclear higher-order structure. Commun. Integr. Biol. 2012. p. 134-139.

[33] Nouspikel T, Hanawalt PC. Terminally differentiated human neurons repair transcribed genes but display attenuated global DNA repair and modulation of repair gene expression. Mol. Cell. Biol. [Internet]. 2000;20:1562-1570. Available from: http://www.pubmedcentral.nih.gov/articlerender.fcgi?artid=85340\&tool=pmcentrez\&rend ertype $=$ abstract .

[34] Hara T, Nakamura K, Matsui M, et al. Suppression of basal autophagy in neural cells causes neurodegenerative disease in mice. Nature. 2006;441:885-889. 
[35] Parkes TL, Elia AJ, Dickinson D, et al. Extension of Drosophila lifespan by overexpression of human SOD1 in motorneurons. Nat. Genet. 1998;19:171-174.

[36] Xiao C, Mileva-Seitz V, Seroude L, et al. Targeting HSP70 to motoneurons protects locomotor activity from hyperthermia in Drosophila. Dev. Neurobiol. 2007;67:438-455.

[37] Sanyal S. Genomic mapping and expression patterns of C380, OK6 and D42 enhancer trap lines in the larval nervous system of Drosophila. Gene Expr. Patterns [Internet]. 2009;9:371-380. Available from: http://linkinghub.elsevier.com/retrieve/pii/S1567133X09000258.

[38] Libert S, Zwiener J, Chu X, et al. Regulation of Drosophila life span by olfaction and food-derived odors. Science (80-. ). [Internet]. 2007;315:1133-1137. Available from: http://www.sciencemag.org/cgi/doi/10.1126/science.1136610.

[39] Jaber N, Dou Z, Chen J-S, et al. Class III PI3K Vps34 plays an essential role in autophagy and in heart and liver function. Proc. Natl. Acad. Sci. [Internet]. 2012;109:2003-2008. Available from: http://www.pubmedcentral.nih.gov/articlerender.fcgi?artid=3277541\&tool=pmcentrez\&re ndertype $=$ abstract

[40] Backer JM. The regulation and function of Class III PI3Ks: novel roles for Vps34.

Biochem. J. [Internet]. 2008;410:1-17. Available from:

http://biochemj.org/lookup/doi/10.1042/BJ20071427. 


\section{Figure Legends}

Figure 1. The 12-h survival after anoxia in the EDTP mutant. (a) \% 12-h survival in heterozygous EDTP mutants $\left(E D T P^{D J 694} /+\right.$, green $)$ and their sibling controls ( $w^{1118}$, grey) to anoxia. Flies at 6-8 days were exposed to 1, 3, or $6 \mathrm{~h}$ anoxia. Left panel, male flies; right panel, female flies; $* * P<0.01$ and $* * * P<0.001$ from two-way ANOVA with Tukey's test. (b) Twoway ANOVA for the analysis of contributing significance of the genotype, anoxia duration and their interaction to the \% 12-h survival.

Figure 2. Improved survivorship after prolonged anoxia in the heterozygous EDTP mutant.

Flies (6-8 days old) were exposed to 0 or $6 \mathrm{~h}$ anoxia. The post-exposure survival curves of flies carrying EDTP mutation (EDTP ${ }^{D J 694} /+$, green) and their sibling controls ( $w^{1118}$, grey) were plotted. Anoxia durations (red text) are indicated. $P$ values are from Log-rank test. Left panels, male flies; right panels, female flies.

Figure 3. Downregulation of EDTP in motoneurons extended lifespan. (a) The survival curves of flies with EDTP knockdown (D42-Gal4/UAS-EDTP-RNAi (III), green) and their controls (D42-Gal4/+, grey; UAS-EDTP-RNAi (III)/+, light blue). (b) The survival curves of flies with EDTP knockdown (UAS-EDTP-RNAi (II)/+; D42-Gal4/+, green) and their controls (D42-Gal4/+, grey; UAS-EDTP-RNAi (II)/+, blue). Two UAS lines carrying independent RNAi constructs, one in the third chromosome and another in the second chromosome, were used. Presented data are from male flies. $* * * P<0.001$ from Log-rank test with "BH" adjustment. 
Figure 4. Downregulation of $E D T P$ in motoneurons improved the survivorship to the protein aggregates expressed in the same cells. (a) The survival curves of flies expressing $\mathrm{A} \beta 42$ (red) and flies with both $\mathrm{A} \beta 42$ expression and EDTP knockdown in the motoneurons (green). $P$ value from a log-rank test. (b) The survival curves of flies expressing polyQ protein aggregates (red) and flies with both polyQ expression and EDTP knockdown in the motoneurons (green). $P$ value from a log-rank test. Notes: D42-Gal4, motoneuron-specific driver; UAS-A $\beta 42$, a UAS line carrying a gene encoding human beta-amyloid peptide $(\mathrm{N}=42)$; UAS-Httex1-Q103eGFP, a UAS line for the expression of polyQ protein aggregates (containing in each molecule a polyglutamine tract with 103 tandem repeats); UAS-EDTP-RNAi, an RNAi line (\#36917) carrying an interference sequence targeting the exon 2 of EDTP. Data are from male flies.

Figure 5. Expression of MTMR14 in the hippocampus and cortex in C57BL/6J and APP/PS1 mice. (a) Immunostaining of MTMR14 in the hippocampus in a C57BL/6J mouse. CA3, CA3 pyramidal neurons; DG, dentate gyrus. (b) Expression of MTMR14 in the hippocampus and cortex in C57BL/6J and APP/PS1 mice. $\alpha$-Tubulin, loading control; * $P<$ 0.05, Student's $t$-test. (c) Expression of MTMR14 in the hippocampus in C57BL/6J mice at different ages (in months). Numbers in the bars indicate the ages of mice.

Figure 6. MTMR14 expression induced by A $\beta 42$ in the rat primary hippocampal neurons and Neuro2a cells. (a) MTMR14 induction by A $\beta 42$ treatment $(0,5$, and $10 \mu \mathrm{M})$ for $72 \mathrm{~h}$ in the rat primary hippocampal neurons. $* * P<0.01$ by one-way ANOVA and Bonferroni's multiple comparisons. (b) MTMR14 expression induced by A $\beta 42$ treatment $(0$ and $5 \mu \mathrm{M})$ for $24 \mathrm{~h}$ in Neuro2a cells. $* * P<0.01$, Student's $t$-test. 


\section{Male}

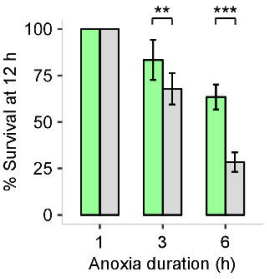

b
Variables

Genotype Anoxia duration

Genotype $\times$ Anoxia duration
Female

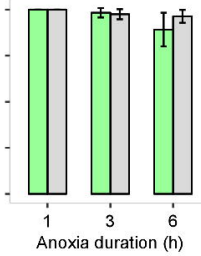

Female

Male

$P=0.126$

$P=0.006$

$\begin{array}{ll}P<0.001 & P=0.126 \\ P<0.001 & P=0.006\end{array}$

$P<0.001$

$P=0.079$ 


\section{Male}

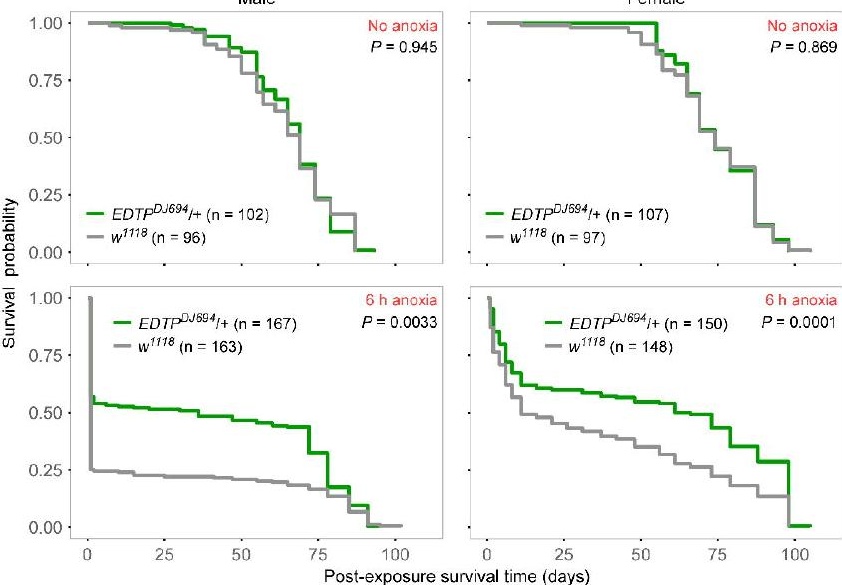


$\left.\left.\begin{array}{l}\text { - UAS-EDTP-RNAi (III)/+ } \\ \text { - D42-Gal4/UAS-EDTP-RNAI (III) }\end{array}\right]_{*}^{*}\right]^{*}$

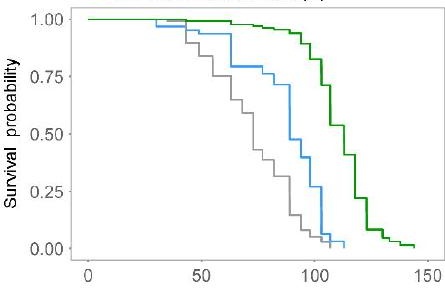

- D42-Gal4/+

$\left.\left.\begin{array}{l}\text { - D42-Gal4/+ } \\ \text { - UAS-EDTP-RNAi (II)/+ } \\ \text { - UAS-EDTP-RNAi (II)/+;D42-Gal4/+ }\end{array}\right]_{*}^{*}\right]_{*}^{*}$

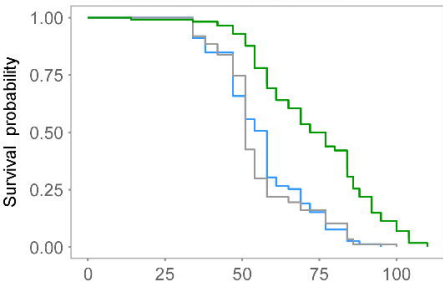

Lifespan (days) 
- UAS-AB42/Y;D42-Gal4/+

- UAS-AB42/Y;;D42-Gal4/UAS-EDTP-RNAi

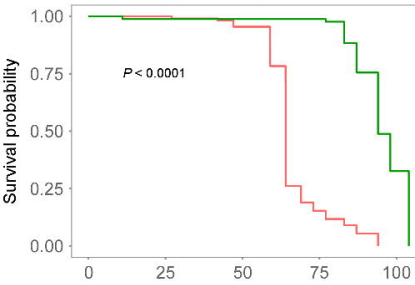

b

- UAS-Httex1-Q103-eGFP/ $/ ;$ D42-Gal4/+

- UAS-Httex1-Q103-eGFP/Y;D42-Gal4/UAS-EDTP-RNAi

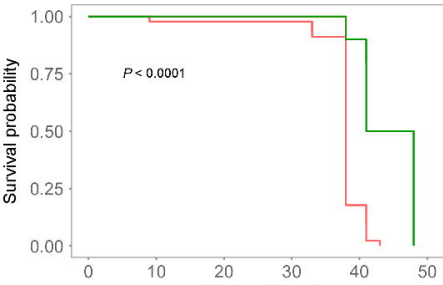

Lifespan (days) 


\section{a}

C57BL/6J

$=\mathrm{CA3}$

DG

I

C

C57BL/6」 APP/PS1

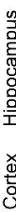

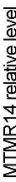

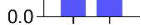

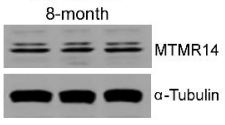

MTMR14

a-Tubulin

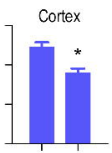

C57BL/6J Hippocampus
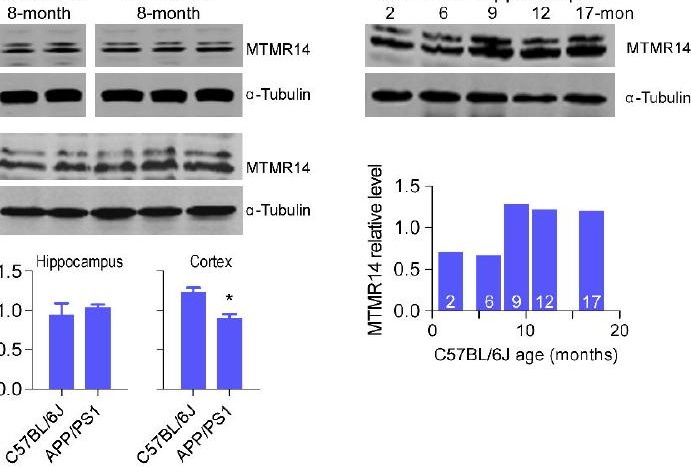

$\alpha$-Tubulin 
a Rat primary hippocampal neurons

Control $\quad A \beta 42,5 \mu M 72 h \quad A \beta 42,10 \mu M 72 h$

MTMR14

a-Tubulin

b

Neuro2a cells

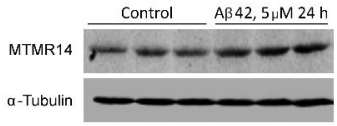

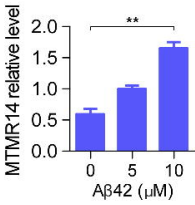

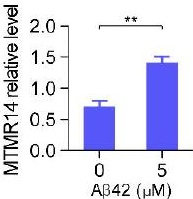

